\title{
Understanding and Prevention of Low Back Pain in Care Workers
}

\author{
Akira MINEMATSU ${ }^{1}$ \\ ${ }^{1}$ Department of Physical Therapy, Faculty of Health Science, Kio University, 4-2-2 Umaminaka, Koryo-cho, Kitakaturagi-gun, \\ Nara 635-0832, Japan
}

\begin{abstract}
The aim of this study was to investigate the actual situation of low back pain (LBP) prevention in care workers with questionnaires, and this data were used to clarify and decrease LBP factors. Subjects were 52 care workers $(30.7 \pm 10.0$ years old) in a nursing home who filled out two kinds of questionnaires about LBP, one about the prevalence of LBP (QN1) and the other about LBP control measures (QN2). They had either license of certified care worker, 1st or 2 nd class care worker. The data were collected for the purpose of finding differences in age and career (QN1) and differences in LBP control measures (QN2) between subjects with and without LBP. The average career of the subjects was $19.1 \pm 12.5$ months, and the prevalence of LBP among them was $46.2 \%$ ( 24 care workers), of which $66.7 \%$ began having LBP after starting work as care workers. Moreover, $75.0 \%$ of these subjects began having LBP within a year after starting work. Further, of the 24 care workers with LBP, $66.7 \%$ reported constantly experiencing LBP. The major risk factors given for LBP in care work were transfer, the replacement of diapers, and movement in a half-sitting posture. Compared with LBP subjects, rates of taking preventative steps in the non-LBP group were low. More than $80 \%$ of the subjects with LBP engaged in prevention methods such as using body mechanics, learning the proper way to perform care activities, using LBP support belts, and increasing their skills and knowledge with regard to their job responsibilities. Most subjects began to engage in such prevention methods after the onset of LBP. The results of this investigation indicated that most subjects did not engage in measures to counter their LBP until after it had already started, and that they selected prevention methods which were easy to perform and effective. It is important for care workers to learn the best ways to inhibit pain and prevent the occurrence, or recurrence, of LBP. It is necessary for physical therapists to grasp the actual situation of LBP prevention as an occupational disease and to educate this.
\end{abstract}

Key words: low back pain, care workers, questionnaires

(J Jpn Phys Ther Assoc 10: 27-31, 2007)

L diseases. The chief risk factors of occupational LBP are heavy physical work, static work posture, lifting and forceful movements, pushing and pulling movements, bending and twisting of the trunk, repetition of the same work, and whole-body vibration ${ }^{1)}$. Depending on these factors, the prevalence of LBP can rise remarkably. Most of these factors are present in the actual working conditions of care workers. Moreover, shortages of care workers and irregular working hours or shifts further influence the risk

Received: June 24, 2005

Accepted: May 20, 2006

e-mail: a.minematsu@kio.ac.jp factors of LBP. LBP can also be caused by factors in the working environment such as the stress of personal relations. In addition, due to increases in LBP medical expenses $^{2}$, it is hard for LBP patients to return to work $^{3)}$. Thus, care workers are exposed to many physical, psychosocial, and work organizational factors, all of which are connected to LBP. In fact, most care workers experience a career-long prevalence of $\left.\mathrm{LBP}^{4}\right)^{5)}$. WHO reported the importance of prevention and measures of LBP in work environment, as LBP was a typical disease which fulfilled not only work-induced but also work-related diseases $^{6}$. Therefore it is very important to investigate the causes of LBP in care workers and to take the proper 
precautions. It is useful to catch the causes and prevention of LBP as an occupational disease in care workers, because physical therapists can remove the risk factor of LBP and educate the precaution suited for LBP patients. This study investigated the causes and prevention measures of LBP in care workers through questionnaires.

\section{Materials and Methods}

Subjects were 52 care workers $(30.7 \pm 10.0$ years old $)$ of a home for the elderly. There were 8 males and 44 females. They received two kinds of questionnaires about LBP from the researcher directly and filled out these questionnaires. First they filled out Questionnaire 1 (QN1), and after mailing out QN1 they filled out Questionnaire 2 (QN2), which was made in accordance with QN1. The contents of these questionnaires are shown in Table 1. The data were collected for the purpose of finding the prevalence of LBP (QN1) and LBP control measures (QN2). After collecting the data, sample number and rate were found item by item.

In a statistical analysis of the collected data, an unpaired t-test was used to find the difference of age and career between subjects with and without LBP. Differences in LBP control measures between subjects with and without LBP were examined by Fisher's exact test. A p-value less than 0.05 was considered significant.

This study was carried out after obtaining the informed consent of all subjects.

\section{Results}

The rate of valid answers was $100 \%$. All of the care workers had learned care knowledge and skills in school, and they recognized the necessity of nursing education. The average career of the subjects was $19.1 \pm 12.5$ months, and the prevalence of LBP in the present study was $46.2 \%$, of which $66.7 \%$ began having LBP after starting work as care workers. Moreover, $75.0 \%$ of these subjects began having LBP within a year after starting work. Regarding the length of time since the onset of LBP, $50.0 \%$ answered less than a year, $16.7 \%$ answered more than a year, and $33.3 \%$ answered more than five years. The age and length of career responses of the LBP group were significantly higher than those of the non-LBP group (Table 2). The degrees of LBP with the visual analogue scale (VAS) were $37.5 \%$ in $0-3,50.0 \%$ in $4-7$, and $12.5 \%$ in $8-10$. Of the 24 care workers, $66.7 \%$ always felt LBP. The factors given for LBP that had begun before becoming a care worker included a decline in muscle strength and flexibility, a static work posture, and a half-sitting posture, whereas the factors given for LBP that had begun after becoming a care worker were lifting and forceful movements such as transfers, bending and twisting of the trunk such as a half-sitting
Table 1. Contents of questionnaire 1 and 2

\section{Questionnaire 1}

Theme: Understanding of low back pain (LBP) in care workers. Type: unregistered and free writing.

Contents

0 . age, sex, care worker's career

1. Do you have LBP now? (yes or no) * if yes;

A. How long have you been LBP?

B. What is the causes of LBP?

C. What is the degree of LBP in visual analogue scale (VAS)?

2. What movement is hard for low back?

3. Do you have any pain except for LBP? (yes or no) * if yes;

A. What part is painful, and is the degree of pain in VAS?

B. What is the causes of the pain?

4. What do you take steps for LBP?

\section{Questionnaire 2}

Theme: Prevention of low back pain (LBP) in care workers.

Type: unregistered and free writing.

Contents

1. Do you have LBP-control measures as follows? (yes or no)

A. using body mechanics.

B. taking proper ways of care.

C. LBP exercise.

D. using LBP supporter.

E. improvement of muscle flexibility and power.

F. taking care in a pair.

G. learning care knowledge and skills.

H. others.

2. When do you begin to take the preventions?

3. Does your LBP lessen after taking the preventions? (yes or no)

4. What is the most important for you to prevent LBP?

Table 2. Prevalence of LBP

\begin{tabular}{lccr}
\hline & $\begin{array}{c}\text { Age } \\
\text { (years) }\end{array}$ & $\begin{array}{c}\text { Career } \\
\text { (months) }\end{array}$ & $\begin{array}{r}\text { Month since } \\
\text { having LBP }\end{array}$ \\
\hline Non LBP & $25.6 \pm 6.2^{*}$ & $14.7 \pm 8.7^{*}$ & \\
LBP & $36.7 \pm 10.4$ & $25.2 \pm 13.7$ & $55.8 \pm 67.6$ \\
Before employed & $44.0 \pm 5.3$ & $32.0 \pm 10.7$ & $138.0 \pm 57.7$ \\
After employed & $33.0 \pm 10.4$ & $21.8 \pm 14.1$ & $14.8 \pm 6.4$ \\
\hline
\end{tabular}

* Significant difference between LBP and non LBP $(\mathrm{p}<0.01)$. (mean \pm S.D.)

posture, lack of exercise, and a lack of proper care skill (Table 3). The greatest risk movements with regard to LBP among the care workers were transfer $(42.1 \%)$, replacement of diapers $(21.1 \%)$, movements in a half-sitting posture (15.8\%), and others (i.e., giving a bath, replacement of bed sheets, and handling of patients) (Table 3). Of the subjects, $61.5 \%$ had other pains in addition to LBP (Table 4 ). For the 
Table 3. The movements caused LBP and hard movements for low back

\begin{tabular}{|c|c|c|c|c|c|}
\hline \multicolumn{4}{|c|}{ Movements caused LBP } & \multirow{2}{*}{\multicolumn{2}{|c|}{ Hard movements for low back }} \\
\hline \multicolumn{2}{|c|}{ LBP before employed } & \multicolumn{2}{|c|}{ LBP after employed } & & \\
\hline Reduce of muscle powe & r $40.0(\%)$ & Transfer & $44.4(\%)$ & Transfer & $45.0(\%)$ \\
\hline Long time sitting & 20.0 & Bath care & 22.2 & Replacement of diapers & 20.0 \\
\hline Half-sitting posture & 20.0 & Lack of exercise & 11.1 & Half-sitting posture & 15.0 \\
\hline Others & 20.0 & Others & 22.3 & Others & 20.0 \\
\hline
\end{tabular}

Table 4. Regions and degrees of pain besides LBP

\begin{tabular}{lrllr}
\hline \multicolumn{2}{l}{ Regions and degrees of pain } & Causes of pain & \\
& (\%) (VAS) & & (\%) \\
\hline Neck & $9.1(2-3)$ & Over load & 37.5 \\
Back & 18.2 & $(2-4)$ & Keeping same posture & 25.0 \\
Shoulder & $27.3(1-5)$ & Being careful of LBP & 12.5 \\
Elbow and wrist & 18.2 & $(1)$ & Others & 25.0 \\
Knee and ankle & $18.2(1-3)$ & & \\
Others & 9.0 & & \\
\hline
\end{tabular}

Table 5. Prevention of LBP

\begin{tabular}{ll}
\hline Acquirement of care knowledge and skills & $33.3(\%)$ \\
Use of body mechanics & 23.8 \\
Improvement of muscle power and flexibility & 14.3 \\
Others & 28.6 \\
\hline
\end{tabular}

VAS: visual analogue scale.

Table 6. Rate of performing LBP-control measures (\%)

\begin{tabular}{lcccccccc}
\hline & $\mathrm{A}$ & $\mathrm{B}$ & $\mathrm{C}$ & $\mathrm{D}$ & $\mathrm{E}$ & $\mathrm{F}$ & $\mathrm{G}$ & $\mathrm{H}$ \\
\hline All subject & 69.2 & 69.2 & 38.5 & 38.5 & 30.8 & 53.8 & 84.6 & 38.5 \\
Non LBP & 57.1 & 57.1 & 33.3 & $0^{*}$ & 28.6 & 42.9 & 85.7 & 28.6 \\
LBP & 83.3 & 83.3 & 37.5 & 83.3 & 33.3 & 66.7 & 83.3 & 50.0
\end{tabular}

A: using body mechanics. B: taking proper ways of care. C: LBP exercise. D: using LBP supporter. E: improvement of muscle flexibility and power. F: taking care in a pair. G: learning care knowledge and skills. H: others.

*: significant difference from LBP $(\mathrm{p}<0.01)$.

prevention of LBP specifically, however, the acquirement of care knowledge and skills (33.3\%), the use of body mechanics $(23.8 \%)$, the improvement of muscle power and flexibility (14.3\%), and other methods (i.e., exercises for LBP, working in pairs, and using supporters for the trunk) were the most common responses given (Table 5).

Compared with the LBP group, all of the rates of utilized LBP prevention methods given by the non-LBP group were low except for Method G (learning care knowledge and skills). In particular, none of the subjects in the non-LBP group performed Method D (Table 6). In total, $53.8 \%$ of the subjects began to utilize the prevention methods after beginning care work, $30.8 \%$ began after the onset of LBP, and the rest began before beginning their jobs as care workers.

\section{Discussion}

LBP is one of the most severe problems faced by care workers, as it can deteriorate one's ability and motivation for work. This study investigated the LBP conditions of care workers and how they understand and deal with the problems caused by LBP. Of the subjects with LBP, $66.7 \%$ began having LBP after beginning employment as care workers. Moreover, $75.0 \%$ of these began having LBP within a year after beginning work. This finding suggests that they might not have been aware of LBP prevention measures. In fact, most of these subjects did not try any of the available prevention measures for LBP until after the onset of LBP. More over, few subjects without LBP took any precautions whatsoever. It is therefore important for care workers to be educated about work-related injuries and for employers to insure that work environments are in good condition.

The causes of LBP that began after beginning work were different from those of LBP that began before starting work. Subjects with LBP before beginning care work gave the reduction of muscle power, extended time in a sitting 
position, and a half-sitting posture as the main reasons for their LBP. On the other hand, regarding LBP that began after starting work, subjects responded that LBP occurred during caregiving and that transfer was the main reason for its onset. Thus, the causes of LBP varied depending on the type of work. Transfer includes such LBP risk factors as lifting and forceful movements, pushing and pulling movements, and the bending and twisting of the trunk. Care workers must engage in transfer and other heavy motions many times in a single day. This result was similar to that reported by Cheung7), stating that back strains that occur while handling clients are a major health care hazard in the nursing profession. In addition, the American Nurses Association (ANA) has promoted a campaign to encourage a health care industry-wide effort to prevent back and other musculoskeletal injuries ${ }^{8}$. Finally, with regard to the current study, lifting was given as the major factor of LBPrelated stress. ANA gave various LBP measures for patients and nurse and these measures are necessary and important. It is considered that the quickest and easiest step in ANA's measures is education and training, because the cost is low and there is a physical therapist at least in hospital and elderly facilities. All care workers must have education and training to care the patients, but many of them cannot make use of the experiences. It is important for physical therapists to enlighten LBP prevention because they are professional of body mechanics.

Rates of reported shoulder pain were the highest in subjects with LBP in this study. Myers et al. ${ }^{9)}$ reported high back and shoulder injury incidence rates among nursing assistants and stressed the importance of social integration and work organization issues. This suggests that shoulder pain is associated with transfer, and that lifting, pulling, and holding movements burden the shoulders. Indeed, not only the shoulders, but also other regions of the body are affected by LBP either directly or indirectly.

Most LBP patients recover within two months of the onset of pain, but some develop chronic $\mathrm{LBP}^{10}$ ), and there are many non-physical factors that can influence the severity of LBP, such as working environment, working time, and working shift, etc. It is necessary, therefore, to try to select the best available LBP-control measures. In the present study, eight methods of LBP prevention were investigated (Table 6). The rates of engagement in LBP prevention measures among LBP subjects were higher than those of non-LBP subjects for all methods except for Method G. As stated above, this indicates that non-LBP subjects were less concerned about LBP prevention, while LBP subjects understood these methods as ways to inhibit or improve the aggravation of LBP rather than to prevent it. The percentages of engagement in Method C (LBP exercise) and Method E (improvement of muscle flexibility and power) were particularly low, despite the fact that $14.3 \%$ of the subjects responded that the improvement of muscle flexibility and power was the most important prevention method. It is difficult, however, to determine the effectiveness of these methods on LBP with regard to the time required for improvement and subject patterns of continuation. In contrast, more than $80 \%$ of the subjects with LBP reported engaging in prevention Method A (using body mechanics), Method B (performing care activities more properly), Method D (using LBP support belts), and Method G (learning care knowledge and skills). Methods A, $\mathrm{B}$ and $\mathrm{G}$ came under education and training, and methods $\mathrm{B}$ applied assistive equipment in ANA's measures. All these methods can be taken at low cost easily. These methods can be utilized while at work and therefore do not require additional time outside of work to be effective. In addition, the biomechanical and postural stresses found in this study correlated with previously reported musculoskeletal injury rates $^{11)}$ and studies that found that the ergonomic design of care work environments can help minimize LBP ${ }^{12) 13)}$. Back supports have also been shown to be effective in the prevention and reduction of $\mathrm{LBP}^{14) 15)}$. These reports show that LBP patients can easily engage in prevention measures and therefore realize their effectiveness.

This investigation demonstrated that most subjects did not actively engage in measures to prevent LBP until after they had LBP. Further, they selected prevention methods which were both easy to perform and effective. Therefore it is important to devise LBP prevention methods that care workers can easily and effectively perform while on the job. The first thing to solve LBP in work environment is to decrease the risk factor of LBP. It was considered that physical therapists could play an important part in introducing, teaching and supporting LBP preventions.

\section{References}

1) Riihimaki H: Low-back pain, its origin and risk indicators. Scand J Work Environ Health 17: 81-90, 1991.

2) Spengler DM, Bigos SJ, et al:: Back injuries in industry: a retrospective study. Overview and costs analysis. Spine 11: 241-245, 1986.

3) Pransky G, Benjamin K, et al:: Work-related outcomes in occupational low back pain: a multidimensional analysis. Spine 27: 864-870, 2002.

4) National Institute for Occupational Safety and Health, USA: Guidelines for protecting the safety and health of health care workers. 1988 ; No.88-119. (http://www.cdc.gov/niosh/88119.html).

5) Omokhodion FO, Umar US, et al.: Prevalence of low back pain among staff in a rural hospital in Nigeria. Occup Med 50: 107-110, 2000.

6) Report of WHO Expert Committee: Identification and control of work-related diseases. Geneva, 1985.

7) Cheung K: Close calls for low-back injuries. Can Nurse 95 : 47-48, 1999.

8) de Castro AB: Handle with care: The American Nurses Association's campaign to address work-related musculoskeletal disorders. Online J Issues Nurs 9: 3, 2004. 
9) Myers D, Silverstein B, et al.: Predictors of shoulder and back injuries in nursing home workers: a prospective study. Am J Ind Med 41: 466-476, 2002.

10) Lehmann TR, Spratt KF, et al:: Predicting long-term disability in low back injured workers presenting to a spine consultant. Spine 18: 174-178, 1993.

11) Stuebbe P, Genaidy A, et al:: The relationships between biomechanical and postural stresses, musculoskeletal injury rates, and perceived body discomfort experienced by industrial workers: a field study. Int J Occup Saf Ergon 8: 259-280, 2002.

12) Snook SH, Cambanelli RA, et al.: A study of three preventive approaches to low back injury. J Occup Med 20: 478-481, 1978.

13) Fragala G, Bailey LP: Addressing occupational strains and sprains: musculoskeletal injuries in hospitals. AAOHN J 51: 252-259, 2003.

14) Kraus JF, Brown KA, et al:: Reduction of acute low back injury by use of back supports. Int J Occup Environ Health 2: 264-273, 1996.

15) Gatty CM, Turner M, et al:: The effectiveness of back pain and injury prevention programs in the workplace. Work 20 : 257-266, 2003. 